\title{
Analysis on the Design Patterns of Cloud Computing in Mobile Learning Systems
}

\author{
Chanjuan Liu \\ Department of Basic Courses, \\ Shandong University of Science and Technology, Tai'an, China \\ echochanjuan@sina.com
}

\begin{abstract}
As a low-cost, highly effective network application model, cloud computing gradually affects peoples' learning, work and life. On the basis of a brief description of cloud computing and its features, this paper focuses on design patterns in mobile learning, and explores the educational environment of cloud computing.
\end{abstract}

Keywords- Cloud Computing ; Mobile Learning; Learning System;Structure Design

\section{INTRODUCTION}

A.Concept of Cloud Computing and Mobile Learning System

Cloud computing is a new term---- the development of advanced parallel computing, distributed computing and Grid Computing; an evolution of mixed concepts of virtualization, utility computing, IaaS (Infrastructure as a Service), PaaS (platform As a Service), SaaS (Software as a Service). According to Wikipedia explanation, cloud computing is a dynamic calculation method for virtualization resources expanding easily via the Internet ,where the user neither need details of internal expertise nor have direct control of infrastructure. From Amazon's "Elastic Compute Cloud" to IBM's "Blue Cloud"; from Google Apps to Microsoft Windows Live ,cloud computing research has become a fashion sought after by the industry and enterprise ;government and education. People saw more new opportunity and joined the ranks of the cloud.

B. Mobile Learning Development Status

Mobile learning refers to the use of wireless mobile communication network technology, a new type of digital learning to obtain educational information, education resources, and education services with help of Communication devices (such as mobile phones, personal digital assistants PDA, the Pocket PC, etc.)

At present, study on mobile learning abroad mainly is divided into two categories from research purposes, one is initiated by E-learning providers used for corporate training; another type initiated by some educational institutions are to improve teaching, learning and management. For example, Uni Wap mobile learning projects in the Helsinki University, Finland, teachers participating are equipped with a WAP phone. Once they get themes of study, they can immediately send their ideas to teaching server. In this way, other members of the training team feel free to browse through the WAP phone without meeting each other.

\section{Function Designs OF MobiLE LEARNING SySTEM BASED ON CLOUD COMPUTING}

Supported by cloud computing to build a functional, flexible and interactive mobile learning system which is easy to use and adaptive to learning device is an urgent demand in cloud computing era A. Cloud-based mobile learning system should have the following functional features:

A.Providing Hierarchical Management and Software Support

The whole system is divided into two parts managed by a professional team in the cloud terminal. The effectiveness of the system is decided by the team. For the clients, the management entrance is focused on a particular subject or a particular field of management. This manager is equivalent to a squad leader or a teacher, this part of the management is to ensure the learning quality of clients.

The cloud provides all the learning software needed for the clients with bare machine, and the software is constructed above the various standards and protocols. When the user needs to use application software, without having to download them or knowledge of the mobile learning system, thus all kinds of people can conduct mobile learning shaking off the many troubles for learners to install, download or upgrade software, make learning going well with high efficiency. The system can also allocate operating system and unified maintenance by the "cloud" for every user avoiding incompatible between the operating system and the learning software without worrying about virus attacks, even paralysis caused by the entire mobile device system .Users do not need to buy expensive mobile devices of better configuration and performance., the most common cell phone does the job. As a result, even the people of economically backward regions can be taught to achieve mobile learning and balanced development.

B.Providing Dynamic Storage Space

Learners can store and withdraw all of your resources in the cloud at any time without worrying about the sufficiency of free storage space, loss of information which not only enrich the learning resources, but also achieve the sharing of resources, to the largest extent $t$ without the limitation of time and space.

C. Providing the Dynamic Data Service

The data includes the original data, semi structured data and the processed structured data. The system must be able to provide intelligent service such as the massive sharing, mining, searching, management, analysis and so on, in which learners will not be "lost". In addition, the system can revise 
and convert format of dynamic teaching content into the information release form supported by terminal for equipment self-adaptation.

D. Providing Ubiquitous Resources

Exploitation of learning resources is no longer confined to certain educational institutions and educators, let alone geographical restrictions. Instead , a team is to do the work, thereby realizing a plurality of resources, to meet the diversified social demands for knowledge .At the same time learning resource is more abundant, and all kinds of learning request is satisfied when the learner's own learning data stored shared in the cloud .

E. Providing the mobile interactive zone

The user can interact with teachers or other learners to view publish articles, video and chat, anywhere by moving the device. When the user is not online, mobile phone short message and instant communication is to notify users to view and their related information. Learners can also create a multi virtual community for the exchange of learning.

\section{ARchitecture Design Based on Cloud Mobile LEARNING SYSTEM}

Microsoft Corp are developing Internet operating system "Midori", completely separate from the Desk top. The emergence of mobile learning model s such as IBM blue cloud computing platform (Blue Cloud) and the Amazon Company's Elastic Compute Cloud), inject fresh blood to the overall cloud Computing system. The structure of constructed a cloud-based mobile learning system Calculation Model, is shown in figure 1 . Figure 1 is a diagram of cloud-based mobile learning system

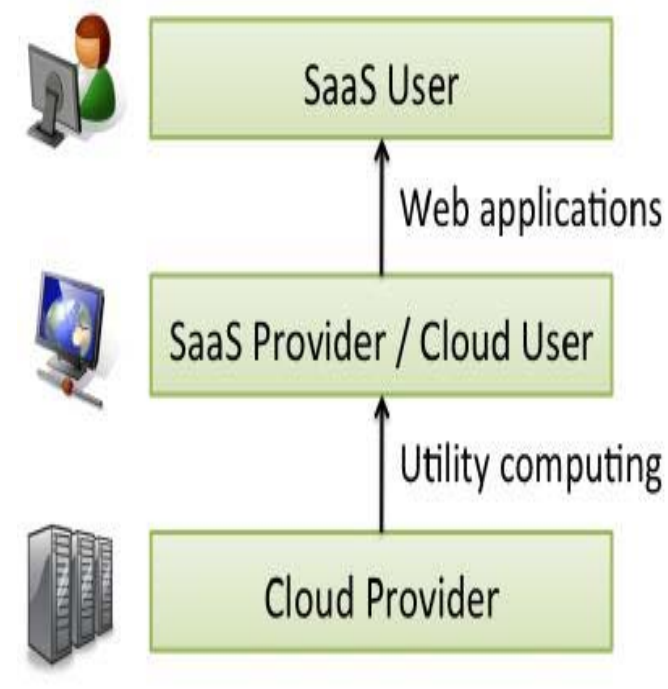

Cloud-based mobile learning system comprises the client, the mobile communications network or $\mathrm{Wi}-\mathrm{Fi}$, based on the network platform and cloud Operator platform of 3-tier application (UI, BLL, DAL).

A.The Client Terminal

Here the client terminal refer to Mobile terminal-a medium of interaction between users and the system which present system content .The supportive device can be mobile phone, PDA and other mobile equipment, which provide different categories of users with different interface and function.

B. Mobile Communication Network or WIFI

Users communicate via mobile terminal like mobile communication network or wireless network in the network platform. The wireless network can be a dedicated network established by some institutions or a public network provided by network operator as long as the provision of wireless and mobile connection reaches a certain band width.

C.Network platform based on three layers

The platform is a system frame, not bearing any specific data, but as the foundation of whole system, its main function is to identify what kind of service the user needs, and then call cloud computing platform to provide related services through the interface. A Presentation layer (UI) which is the interactive interface, between user and the system responsible for the user's session handling. It runs in the browser program mainly through mobile devices, to achieve users' communication with Business logic layer (BLL) or even the operational database access layer (DAL) which is responsible for handling application requests from layer to carry on the logic business process. The analysis and treatment will be submitted to the cloud computing platform directly through the interface program

The platform design comprises five modules: the management module, communication module, learning module, storage module and the application software module.

1) Management modules: the management module manage the basic information of the user, complete registration and settings and management of users' right. The system has three user types: students, teachers, and system administrators.

2) Learning modules: learning module is the main module which not only provides learners with a selflearning and self-managed space, but also supports teachers to track learning progress and knowledge of learners to grasp the situation. This module provides a variety of types of learning for learners of different character to choose from. These types of learning are: message type , game and virtual community type, browse, links, multimedia presentation type.

3) AC module: to provide Information exchange environment between teachers and learners, learners with learners, teachers with teachers. It is an important way for the learners to engage in academic discussions, to exchange learning experience through the Forum, Blog , WIKI,Twitter for real-time and non-real-time cross-learning on various content and themes. In addition, this module also set a $Q$ \&A Area.

4 )Storage module: This module provides a virtual hard disk function, enabling the learners to study at any time anywhere with the use of their own learning materials or sharing information with others. Users need to classify their stored resources, and make identification icon to facilitate the memory.

5)Application software module: This module is to solve problems like :the mobile terminal can not run some 
learning software, time-consuming for installation and maintenance of software. Softwares are divided into a number of functional areas, such as: word processing software area, the graphics processing software district. Users can find and click, then use software they need , and the software can be used not only to view the content, but to do online editing.

\section{ClOUd COMPUTING PlatFORM}

In the design of mobile learning system many factors such as: learning outcomes, learning resources, learning objects, learning cost need to be considered. This cloud computing platform is a core part of the system, as well as the final function implementation module of the entire system.. Services needed by the user interface with the cloud computing platform, for processing which is completed by the interface data in turn and then pass out to the user. It includes three major modules: data storage, calculation and management. Calculation Module split at the user's computing tasks, and then distributed parallel computing with the corresponding function node to the cloud. After the calculation, result collection, consolidation (such as sorting, merging Etc.) are to be returned to the user. Management module achieve self-management and selftuning data to facilitate query search. Storage module based on the three-tier structure of the network platform should be divided into five sub-parts library modules: user information library, library of learning resources, exchange library, repository, software to store information

\section{CONCLUSION}

Currently, mobile learning supported by cloud computing technology is still in the initial exploratory stage, but its unique advantages heralded application potential of cloud computing in the field of mobile learning .This paper explored the cloud-based mobile learning system design, to achieve a combination of new technology and advanced learning concept to meet personalized, diversified learning of everyone in the information society anytime, anywhere to improve the quality of education, to achieve a balanced development of education and nurture more high-quality talents by providing a new strategy for training of education. In spite that many problems exist in construction of cloud computing mobile learning system, with the maturing calculation technique and applications ,the mobile learning system supported by cloud computing technology is bound to demonstrate and expand strong vitality in the near future, and have a profound impact on educational leadership domain.

\section{ACKNOWLEDGMENT}

This work is supported by Qunxing Project of Shandong University of Science and Technology NUMBER ( qx102143)

\section{REFERENCES}

[1] Chengli Ye,Fumeng Xu. Basics of mobile learning[J].Open Educational Research,2004,(3):23-26

[2] Peng Liu, Yao Shi, San-li Li, Computing Pool--a Simplified and Practical Computational Grid Model, the Second International Workshop on Grid and Cooperative Computing (GCC 2003), Shanghai, Dec 7-10, 2003, published in Lecture Notes in Computer Science (LNCS), Vol. 3032, Heidelberg: Springer-Verlag, 2004.

[3] Ya Qin Zhang ,Dance with th Cloud.-Comments onCloud Computing[Z], 《INSIGHT》,

[4] Peng Liu, Yao Shi, San-li Li, Computing Pool--a Simplified and Practical Computational Grid Model, the Second International Workshop on Grid and Cooperative Computing (GCC 2003), Shanghai, Dec 7-10, 2003, published in Lecture Notes in Computer Science (LNCS), Vol. 3032, Heidelberg: Springer-Verlag, 2004. 